\title{
Detection Methods for Early Caries Diagnosis: A Systematic Review and Meta-Analysis
}

\author{
Petros Foros $^{a}$ Elissaios Oikonomou ${ }^{a}$ Despina Koletsi ${ }^{b}$ Christos Rahiotis ${ }^{c}$ \\ ${ }^{a}$ School of Dentistry, National and Kapodistrian University of Athens, Athens, Greece; ${ }^{b}$ Clinic of Orthodontics and \\ Pediatric Dentistry, Center of Dental Medicine, University of Zurich, Zurich, Switzerland; ' ${ }^{\mathrm{D}}$ Department of Operative \\ Dentistry, School of Dentistry, National and Kapodistrian University of Athens, Athens, Greece
}

\section{Keywords}

Caries detection · Early caries · Incipient caries · Permanent teeth · Primary teeth

\begin{abstract}
The aim was to appraise the evidence on the performance of various means for the detection of incipient caries in vivo. Five databases of published and unpublished research were searched for studies from January 2000 to October 2019. Search terms included "early caries" and "caries detection." Inclusion criteria involved diagnostic test accuracy studies for early caries detection in permanent and primary teeth. A risk-of-bias assessment was performed using the QUADAS-2 tool. We performed the study selection, data extraction, and risk-of-bias assessment in duplicate. The review protocol was a priori registered in the Open Science Framework. Of the initially 22,964 search results, 51 articles were included. For permanent teeth, when histologic examination was considered as the reference for occlusal surfaces, the sensitivity (Se) range appeared high for the DIAGNOdent Pen (DD Pen) at $0.81-0.89$, followed by ICDAS-II at $0.62-1$, DIAGNOdent (DD) at $0.48-1$, and bitewing radiography (BW) at $0-0.29$. The corresponding specificity $(\mathrm{Sp})$ range was: DD Pen 0.71-0.8,
\end{abstract}

ICDAS-II 0.5-0.84, DD 0.54-1, and BW 0.96-1. When operative intervention served as the reference for occlusal surfaces, again, the DD means valued the most promising results on Se: DD $0.7-0.96$ and DD Pen $0.55-0.90$, followed by ICDAS-II 0.25-0.93, and BW 0-0.83. The Sp range was: DD 0.541, DD Pen 0.71-1, ICDAS-II 0.44-1, and BW 0.6-1. For approximal surfaces, the Se was: BW 0.75-0.83, DD Pen 0.6, and ICDAS-II 0.54; the Sp was: BW 0.6-0.9, DD Pen 0.2, and ICDAS-II 1. For primary teeth, under the reference of histologic assessment, the Se range for occlusal surfaces was: DD $0.55-1$, DD Pen 0.63-1, ICDAS-II 0.42-1, and BW 0.31-0.96; the respective Sp was: DD 0.5-1, DD Pen 0.44-1, ICDAS-II 0.61-1, and BW 0.79-0.98. For approximal surfaces, the Se range was: DD Pen 0.58-0.63, ICDAS-II 0.42-0.55, and BW 0.14-0.71. The corresponding Sp range was: DD Pen 0.85-0.87, ICDAS-II $0.73-0.93$, and BW 0.79-0.98. Se and Sp values varied, due to the heterogeneity regarding the setting of individual studies. Evidently, robust conclusions cannot be drawn, and different diagnostic means should be used as adjuncts to clinical examination. In permanent teeth, visual examination may be enhanced by DD on occlusal surfaces and BW on approximal surfaces. In primary teeth, DD Pen may serve as a supplementary tool across all surfaces. @ @ $2021 \mathrm{~S}$. Karger AG, Basel
Correspondence to:

Christos Rahiotis, craxioti@dent.uoa.gr 


\section{Introduction}

Caries prevalence has been declining over the last decades [Jones et al., 2017]. However, the burden of the disease is still profoundly evident and impacting, as it afflicts most people to some extent, despite the growing awareness on the benefits of oral hygiene and the use of fluoride, especially regarding specific types of teeth and surfaces [Ekstrand and Qvist 2015]. Still, in 2015, >10\% of the world's population has been affected by dental caries, underpinning the pervasiveness of this disease across all age groups, and substantiating its pronounced social impact [Vos et al., 2016]. Caries may not only account for decayed teeth, but also for days of pain, sleepless nights, and time lost from school and work [Goodwin et al., 2015; Kassebaum et al., 2015].

An important factor contributing to the high prevalence is that caries may progress undetected in the initial stages and later develop into cavities. Therefore, research in the field, should be focused on the early and accurate detection of incipient caries. Conditional to this, caries prevalence and its socio-economic cost might be subsequently reduced and, concurrently, the basic principle of minimally invasive dentistry would be reinforced.

The most common diagnostic tools for caries are clinical examination and radiography [Gomez 2015; Lino et al., 2015; Diniz et al., 2016; Lenzi et al., 2016]. Nevertheless, these methods are not without shortcomings. First, monitoring caries progression by direct visual examination is particularly challenging, due to the low reproducibility [Hintze et al., 1998]. Dentists are not able to reliably determine the subtle visual and tactile differences between active and inactive enamel lesions from a single clinical examination [Ekstrand et al., 2005], as caries is a dynamic and fluctuating process involving demineralization and remineralization over a period of time [Fejerskov 1997]. Furthermore, radiography may underestimate the depth of the carious lesion and present a very low sensitivity rate, especially in the detection of incipient caries [Souza et al., 2013; Menem et al., 2017]. Last, the clinical criteria for caries classification vary across different systems.

Considering the above, new diagnostic tools with different characteristics have attained increased interest in the dental industry in the recent years, effectively contributing to early caries detection. These methods have been generally reported to present high sensitivity (Se) and good reproducibility but demonstrate low specificity (Sp) and some are affected by potential confounding factors [Gomez, 2015]. The National Institutes of Health (NIH)
Consensus Development Conference on Diagnosis and Management of Dental Caries through Life in 2001 reached the conclusion that the evidence base for the currently applicable methods for the detection and activity assessment of non-cavitated carious lesions was not adequate to substantiate any formal recommendation to adopt them [Bader et al., 2002].

There is ambiguity regarding the net effectiveness of diagnostic methods, as conventional ones are more specific than sensitive, while newer methods are considered more sensitive than specific [Gimenez et al., 2015]. In this context, the subtle balance between Se and Sp of each diagnostic method might influence the selected treatment option and clinical decisions, and leading, potentially, to misclassification followed by subsequent over-treatment or under-treatment [Zaidi et al., 2016].

Heterogeneity, unconfirmed data, and subjectivity according to diagnostic tools in the current published research all fuel future initiatives to comprehensively assess the existing evidence in the field. Systematic reviews and meta-analyses comprise the most powerful research tools to identify and rate the best available data. The aim of this timely systematic review of diagnostic test accuracy was to compare all in vivo studies, by referring to the accuracy of the diagnostic means available to clinicians and frame future perspectives on the most efficient methods for early caries detection in the permanent and primary dentition.

\section{Materials and Methods}

\section{Protocol}

The aim of this review was to answer the question: "What is the accuracy of different diagnostic tools for early caries detection in primary and permanent teeth, as reported through visual examination, radiography, fluorescence, ultrasonic detection, infrared spectroscopy, and others, compared against reference standards for caries detection (histological, microscopic, and operative procedures) regardless of the type of teeth, dentition and age of the patient population?"

The protocol of the Cochrane Collaboration (2014; The Nordic Cochrane Centre, Copenhagen, Denmark) was followed in this review with Review Manager (RevMan v5.3.). The reporting scheme of the review was aligned to the recommendations of the latest PRISMA statement for diagnostic tests accuracy studies [McInnes et al., 2018] and the PICO framework [Schardt et al., 2007]. The review protocol was a priori registered in the Open Science Framework (DOI 10.17605/OSF.IO/X9UWY).

\section{Search Strategy}

An electronic search was conducted of published and unpublished literature, independently and by two investigators (P.F. and E.O.). The main formal databases utilized in this study were MED- 
LINE (via PubMed) and Scopus. Filters were used to ensure that the date range was 2000/01/01-2019/10/25 for MEDLINE and 20002019 for Scopus. Reports in the "grey literature," defined as theses, dissertations, product reports, and unpublished studies via ClinicalTrials.com, Open Grey, and the ISRCTN registry, were also included. The date range for these search engines was 2000/01/01-2019/02/21. A hand search was also conducted of the articles retrieved for full-text evaluation for any additional potential for inclusion.

The main keywords that we used were: "caries diagnosis," "incipient caries," "caries detection," "early caries diagnosis," "technological devices for caries diagnosis," and "caries diagnosis methods.

\section{Eligibility Criteria}

Study Design. Clinical (in vivo) studies referring to the accuracy of different (any) diagnostic tools for the evaluation of caries.

Type of Teeth. No restriction. Studies referring to primary/permanent teeth were included.

Index Tests. Visual, visual/tactile examination, radiography, laser fluorescence, fibre-optic trans-illumination (FOTI), near-infrared spectroscopy (NIR), ultra-sonic caries detector (USD), optical coherence tomography (OCT), and electrical conductance (EC) were assessed.

Target Condition. Any study investigating caries.

Inclusion Criteria. Only studies reporting on Se and Sp values were included.

Exclusion Criteria. Studies not reporting on specific diagnostic methods and that were not in vivo studies were excluded. Animal studies were excluded. Studies that were not written in the English language were not considered.

\section{Study Selection}

Studies gathered from the databases were cross-checked to exclude duplicates. Titles and abstracts were evaluated separately by two reviewers (P.F. and E.O.), according to the study's main topics, with further exploration of the full text. Each reviewer forwarded the studies for inclusion or exclusion, according to the eligibility criteria (kappa $=0.93)$. Discrepancies were discussed among reviewers until a consensus was confirmed. A third reviewer (C.R.) was consulted when needed.

\section{Data Collection Process}

Data were extracted and recorded in a standard form (Zotero v5.0.47, Corp. for Digital Scholarship, and the Roy Rosenzweig Center for History and New Media). This form included specific characteristics of the study (type, title, authors, abstract, publication, volume, issue, pages, date, series, series title, series text, journal abbreviation, language, DOI, URL, ISSN, short title, mean of access, archive, location in the archive, library catalogue, call number, date added, date modified). Data were extracted by two of the reviewers (P.F. and E.O.) and re-examined by another reviewer (C.R.). Inconsistencies were discussed until a consensus was reached.

\section{Quality Assessment of Accuracy Studies}

The methodological quality of the studies was determined by means of the QUADAS-2 (Quality Assessment of Diagnostic Accuracy Studies-2) tool [Whiting et al., 2011]. Four domains were considered to determine the risk of bias and level of concern according to the applicability of the studies:
1 Patient selection: studies with a non-random or non-consecutive sample of patients, with the inclusion of entire cases of cavitated lesions or exclusion of cases difficult to diagnose, were judged as having a high risk of bias concerning the patient selection. This was also the case for case-control studies.

2 Index test: when a threshold was not reported and when diagnostic methods were interpreted with knowledge of the results of the reference standard, the index test domain was classified as being high-risk.

3 Reference standard: when reference standards were performed without knowledge of the index test results, the domain reference standard was classified as having a low risk of bias. All studies in which one of the index tests was also the reference standard were excluded. Moreover, studies that did not report a reference standard were excluded a priori.

4 Flow and timing: when the reference standard was not used for all samples, or if not all samples were included in the analysis, the flow and time domain was classified as having a high risk of bias. Furthermore, when a long period had elapsed (i.e., >1 month) between the index test and the reference standard, the flow and time domain was classified as high-risk because this situation could have resulted in changes in the lesion. The same applied if the interval was not reported.

Concerns about the applicability of the studies were determined as follows:

1 Patient selection: studies implemented with the inclusion of only cavitated lesions and studies with a small sample size $(<20$ lesions) were characterized as having a high concern regarding applicability.

2 Index test: when the index test implementation was different from the review question, a high concern was documented for the specific study.

3 Reference standard: when the gold standard demonstrated the presence of carious lesions in the enamel and/or dentin by histological/microscopic analysis and/or hardness measurement, the concern related to applicability was characterized as low. Studies assessing the validation of the target condition by radiographic analysis or that used hard-tissue removal (drilling) and/or clinical examination (visual-tactile) as their gold standard scored as being of high concern regarding applicability.

\section{Summary Measures and Data Synthesis}

Studies were analyzed and evaluated regarding content validity. Those that met the highest number of criteria needed were judged as having greater content validity. Finally, a qualitative and quantitative analysis of the study outcomes was performed.

Quantitative syntheses of the studies' findings were performed, if applicable, and after exploring heterogeneity levels across individual reports. Random-effects meta-analyses were conducted to account for within- and between-study heterogeneity as well as for correlation between the Se and Sp estimates. The model fits a 2-level mixed regression approach, with independent binomial distributions for the true-positives and true-negatives, and a bivariate approach for the log odds ratio transforms for Se and Sp between studies. Summary estimates of Se and $\mathrm{Sp}$ and diagnostic odds ratio (DOR) with respective confidence interval (CI) and hierarchical summary receiver operating characteristic (HSROC) curves (as curvilinear lines passing through the summary points) were planned, to be presented for all applicable comparisons. Pooled estimates were presented if $\geq 4$ studies were deemed eligible for a 


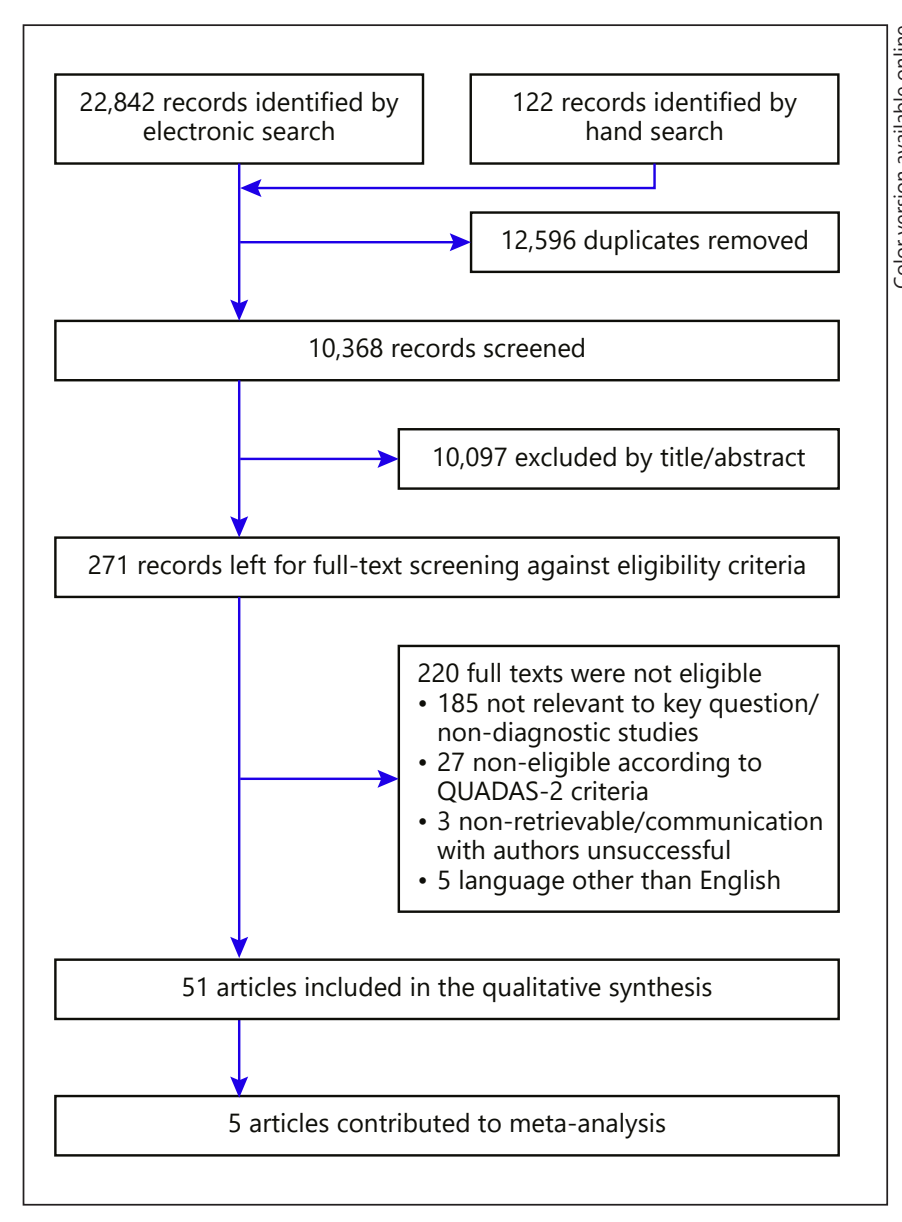

Fig. 1. PRISMA flowchart.

single comparison. Authors were contacted to request data if not all available information was provided within the published document.

\section{Additional Analyses}

Meta-regression analytical techniques were planned for the assessment of the effect of tooth type (permanent or primary) on the pooled estimate. In addition, sensitivity analyses were planned, with the exclusion of high risk-of-bias studies from the syntheses if both high and lower risk-of-bias studies were included, to isolate and explore the effect of the high risk-of-bias studies. Publication bias was planned to be examined through standard funnel plots if $>10$ studies were included in the quantitative syntheses.

\section{Results}

\section{Descriptive Findings and Quality Assessment}

After electronic database and hand-searching, out of a total of 10,368 unique records screened, 271 studies were left for a full-text assessment according to the eligibility

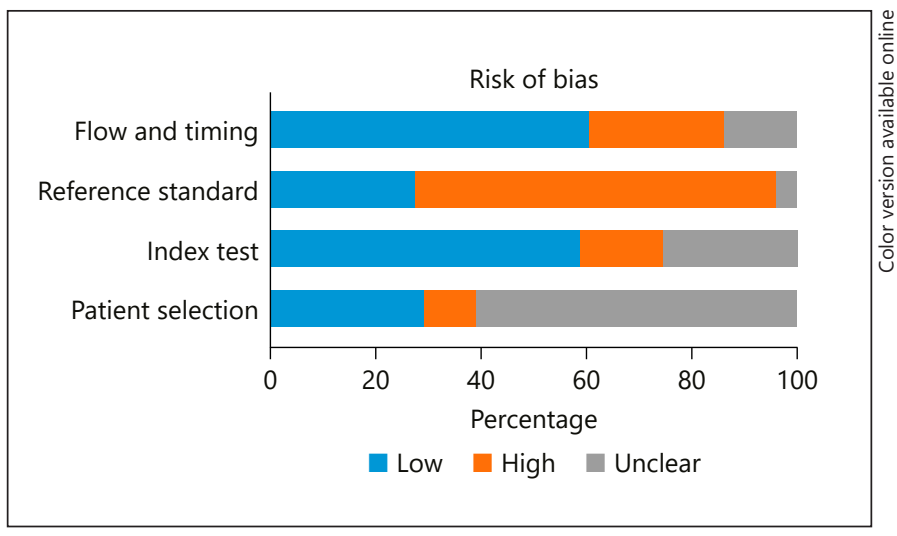

Fig. 2. Proportion of studies with low, high, or unclear risk of bias (\%).

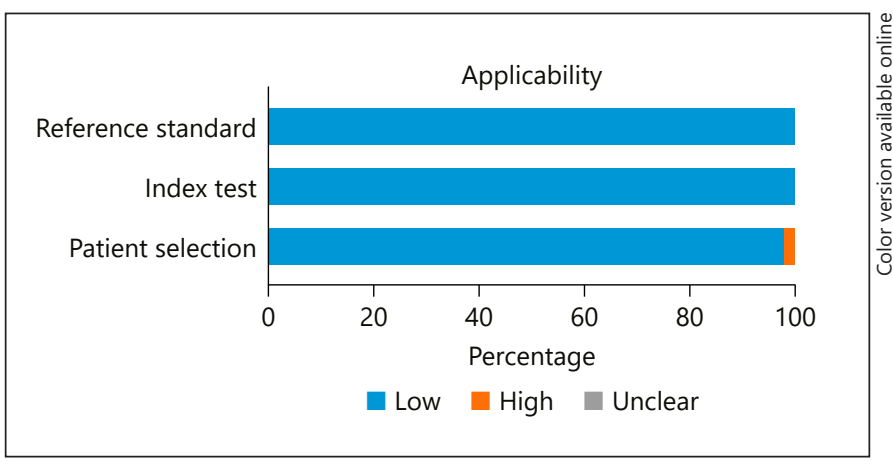

Fig. 3. Proportion of studies with low, high, or unclear concerns regarding applicability (\%).

criteria. Out of these, 51 were finally included and 5 were eligible for the quantitative syntheses overall (Fig. 1). A customized e-mail was sent to the corresponding authors of 27 studies deemed eligible for data request, but only 5 [Heinrich-Weltzien et al., 2002; Reis et al., 2006; Abalos et al., 2009; Diniz et al., 2012; Tassoker et al., 2020] provided relevant additional data. Study characteristics and qualitative data are presented in detail in online supplementary Table 1 (for all online suppl. material, see www. karger.com/doi/10.1159/000516084).

Almost half of the included studies (22/51) were published between 2015 and 2019; the breakdown of the rest, regarding the year of publication was: 14/51 were published between 2010 and 2014, 12/51 in 2005-2009, and only a fraction of $3 / 51$ between 2000 and 2004 .

Results of the risk-of-bias assessment and applicability appear in Figures 2 and 3. Generally, of the 51 included articles, 43 can be classified as having a high risk of bias, 2 a low risk of bias and 6 an unclear risk of bias. Sixty percent of the included studies presented a low risk of bias 
according to flow and timing as well as according to the index test domain. In addition, more than two-thirds $(60 \%)$ of the studies were characterized as having a high risk of bias regarding the reference standard, and another $2 / 3$ were also considered to have an unclear risk of bias when referring to the patient selection domain. Almost all the included studies were characterized as having a low risk of concern regarding applicability in the reference standard, index test, and patient selection domains. The global risk of bias and the overall applicability concerns of the studies appear in Table 1.

\section{Visual Criteria}

Visual criteria were evaluated in 35 studies. ICDAS-II was the most common method for visual examination, with a total of 14 studies (40.0\%) assessing its effectiveness as a diagnostic tool. Ekstrand's criteria were assessed in 10 studies and Nyvad's criteria in 4 .

The sample sizes reported were in the considerably wide range of 25-4,112 teeth. On average, there were two examiners per study, with only a few studies utilizing a different number. Various reference standards were used for validation, with histological examination and operative intervention being, by far, the most used, with application in 15 and 14 studies, respectively. The Se scores of visual methods ranged from 0.04 to 1 and the Sp scores from 0.44 to 1 . Area under the ROC curve data $(\mathrm{Az})$ ranged from 0.56 to 0.99 (Tables 2, 3; online suppl. Tables 2-4).

\section{Radiography}

Intraoral bitewing radiography (BW) was evaluated in 28 studies. The range of the sample sizes was the same as the respective range of studies evaluating visual criteria (254,112 teeth) since some studies simultaneously investigated radiography and visual examination as different index tests.

On average, there were two examiners per study. Histological examination and operative intervention were again the most used reference standards in 11 (39.3\%) and 10 studies (35.7\%), respectively. Se ranged from 0 to 0.96, Sp from 0.6 to 1, and Az from 0.51 to 0.95 (Tables 2, 3; online suppl. Tables 2-4).

\section{DIAGNOdent 2095}

The first-generation DIAGNOdent laser fluorescence device (DD), also known as DIAGNOdent 2095, was evaluated in 18 studies. Sample sizes ranged from 30 to 433 teeth. However, this range was considerably smaller than the corresponding ranges for visual methods and intraoral radiographs. On average, there were two examiners per study. Like the previous methods, operative inter-
Table 1. Risk-of-bias applicability concerns

\begin{tabular}{|c|c|c|}
\hline Study & $\begin{array}{l}\text { Risk of } \\
\text { bias }\end{array}$ & $\begin{array}{l}\text { Applicability } \\
\text { concerns }\end{array}$ \\
\hline Bizhang et al., 2016 & high & low \\
\hline Kim et al., 2017 & high & low \\
\hline Jablonski-Momeni et al., 2014 & high & low \\
\hline Goel et al., 2009 & high & low \\
\hline Reis et al., 2006 & high & low \\
\hline Chan et al., 2018 & high & low \\
\hline Abalos et al., 2009 & high & low \\
\hline Bahrololoomi et al., 2015 & high & low \\
\hline Bozdemir et al., 2013 & high & low \\
\hline Chu et al., 2010 & high & low \\
\hline Costa et al., 2008 & high & low \\
\hline Heinrich-Weltzien et al., 2002 & high & unclear \\
\hline Kavvadia and Lagouvardos, 2008 & high & low \\
\hline Krause et al., 2007 & high & low \\
\hline Lussi et al., 2001 & high & low \\
\hline Matalon et al., 2007 & high & low \\
\hline Melo et al., 2015 & high & low \\
\hline Ozkan and Guzel, 2017 & high & low \\
\hline Huth et al., 2008 & high & low \\
\hline Kavvadia et al., 2018 & high & low \\
\hline Huth et al., 2010 & high & low \\
\hline Ribeiro et al., 2015 & high & low \\
\hline Newman et al., 2009 & high & low \\
\hline Barbería et al., 2008 & high & low \\
\hline Błażejewska et al., 2016 & high & low \\
\hline de Souza et al., 2018 & high & low \\
\hline Holtzman et al., 2015 & high & low \\
\hline Jung et al., 2018 & high & low \\
\hline Mortensen et al., 2018 & high & low \\
\hline Rechmann et al., 2012 & high & low \\
\hline Theocharopoulou et al., 2015 & high & low \\
\hline Akbari et al., 2012 & high & low \\
\hline Baelum et al., 2012 & high & low \\
\hline Bussaneli et al., 2015 & high & low \\
\hline Coutinho et al., 2014 & high & low \\
\hline Novaes et al., 2009 & high & low \\
\hline Zeitouny et al., 2014 & high & low \\
\hline Braga et al., 2010 & high & low \\
\hline Cinar et al., 2013 & unclear & low \\
\hline Diniz et al., 2012 & low & low \\
\hline Freitas et al., 2016 & high & low \\
\hline Kockanat et al., 2017 & high & low \\
\hline Kucukyilmaz et al., 2015 & unclear & low \\
\hline Simon et al., 2016 & high & low \\
\hline Singh et al., 2016 & unclear & low \\
\hline Subka et al., 2019 & unclear & low \\
\hline Tassoker et al., 2020 & high & low \\
\hline Teo et al., 2014 & unclear & low \\
\hline Castilho et al., 2016 & low & low \\
\hline Angnes et al., 2005 & unclear & low \\
\hline Rocha et al., 2003 & unclear & low \\
\hline
\end{tabular}


Table 2. Overall ranges of the basic diagnostic methods

\begin{tabular}{lcccc}
\hline Diagnostic technique & Studies, $n$ & Sensitivity & Specificity & AUC of ROC value \\
\hline Visual examination methods & 35 & $0.04-1$ & $0.44-1$ & $0.56-0.99$ \\
Bitewing radiography & 28 & $0-0.96$ & $0.6-1$ & $0.51-0.95$ \\
DIAGNOdent & 18 & $0.43-1$ & $0.5-1$ & $0.55-0.94$ \\
DIAGNOdent Pen & 20 & $0.16-1$ & $0.2-1$ & $0.4-0.95$ \\
VistaProof & 4 & $0.26-0.92$ & $0.41-0.98$ & $0.66-0.97$ \\
CarieScan Pro & 5 & $0.3-0.92$ & $0-0.97$ & $0.47-0.97$ \\
SoproLife & 4 & $0.86-0.98$ & $0.55-0.96$ & $0.89-0.98$ \\
\hline
\end{tabular}

Table 3. Ranges of the basic diagnostic methods according to the type of surface and dentition (note that studies with mixed dentition do not appear in this table)

\begin{tabular}{|c|c|c|c|c|c|c|}
\hline Diagnostic technique & & & Studies, $n$ & Sensitivity & Specificity & AUC of ROC value \\
\hline \multirow[t]{3}{*}{$\begin{array}{l}\text { Visual examination } \\
\text { methods }\end{array}$} & occlusal & $\begin{array}{l}\text { primary } \\
\text { permanent }\end{array}$ & $\begin{array}{r}8 \\
17\end{array}$ & $\begin{array}{l}0.42-1 \\
0.25-1\end{array}$ & $\begin{array}{l}0.51-1 \\
0.44-1\end{array}$ & $\begin{array}{l}0.77-0.98 \\
0.56-0.96\end{array}$ \\
\hline & approximal & $\begin{array}{l}\text { primary } \\
\text { permanent }\end{array}$ & $\begin{array}{l}4 \\
5\end{array}$ & $\begin{array}{l}0.05-0.96 \\
0.04-0.81\end{array}$ & $\begin{array}{l}0.73-1 \\
0.83-1\end{array}$ & $\begin{array}{l}0.58-0.95 \\
0.77-0.99\end{array}$ \\
\hline & buccal & permanent & 1 & $0.7-0.88$ & $0.97-0.99$ & $0.77-0.99$ \\
\hline Bitewing radiography & approximal & $\begin{array}{l}\text { primary } \\
\text { permanent }\end{array}$ & $\begin{array}{l}8 \\
3\end{array}$ & $\begin{array}{l}0.14-0.96 \\
0.15-0.83\end{array}$ & $\begin{array}{l}0.62-1 \\
0.6-0.99\end{array}$ & $\begin{array}{l}0.58-0.92 \\
0.71\end{array}$ \\
\hline DIAGNOdent & occlusal & $\begin{array}{l}\text { primary } \\
\text { permanent }\end{array}$ & $\begin{array}{r}3 \\
15\end{array}$ & $\begin{array}{l}0.43-1 \\
0.48-1\end{array}$ & $\begin{array}{r}0.5-1 \\
0.54-1\end{array}$ & $\begin{array}{l}\text { not available } \\
0.55-0.94\end{array}$ \\
\hline \multirow[t]{3}{*}{ DIAGNOdent Pen } & occlusal & $\begin{array}{l}\text { primary } \\
\text { permanent }\end{array}$ & $\begin{array}{l}3 \\
7\end{array}$ & $\begin{array}{l}0.63-1 \\
0.6-0.97\end{array}$ & $\begin{array}{l}0.44-1 \\
0.54-0.85\end{array}$ & $\begin{array}{l}0.66-0.95 \\
0.67-0.92\end{array}$ \\
\hline & approximal & $\begin{array}{l}\text { primary } \\
\text { permanent }\end{array}$ & $\begin{array}{l}5 \\
3\end{array}$ & $\begin{array}{r}0.16-0.97 \\
0.6-0.88\end{array}$ & $\begin{array}{l}0.68-1 \\
0.2-0.98\end{array}$ & $\begin{array}{c}0.57-0.92 \\
0.4-0.9\end{array}$ \\
\hline & buccal & permanent & 1 & $0.32-0.78$ & $0.64-0.85$ & $0.65-0.84$ \\
\hline VistaProof & $\begin{array}{l}\text { occlusal } \\
\text { buccal }\end{array}$ & $\begin{array}{l}\text { permanent } \\
\text { permanent }\end{array}$ & $\begin{array}{l}3 \\
1\end{array}$ & $\begin{array}{l}0.26-0.92 \\
0.74-0.85\end{array}$ & $\begin{array}{l}0.41-0.98 \\
0.49-0.80\end{array}$ & $\begin{array}{l}0.72-0.97 \\
0.66-0.80\end{array}$ \\
\hline CarieScan Pro & occlusal & $\begin{array}{l}\text { primary } \\
\text { permanent }\end{array}$ & $\begin{array}{l}3 \\
2\end{array}$ & $\begin{array}{r}0.72-0.91 \\
0.3-0.92\end{array}$ & $\begin{array}{r}0-0.92 \\
0.75-0.97\end{array}$ & $\begin{array}{l}0.47-0.92 \\
0.973\end{array}$ \\
\hline
\end{tabular}

vention and histological validation were the most used reference standards, in 8 and 8 studies, respectively. Se of the DD ranged from 0.48 to $1, \mathrm{Sp}$ from 0.5 to 1 , and $\mathrm{Az}$ from 0.55 to 0.94 . Finally, there was an apparent heterogeneity in the calibration cut-off points of the device (Tables 2, 3; online suppl. Tables 2-4).

\section{DIAGNOdent Pen}

The second-generation DIAGNOdent laser fluorescence device, better known as the DIAGNOdent Pen (DD Pen) was assessed in 20 studies. The number of teeth under evaluation ranged from 37 to 621 , and again, two examiners per study were reported on average. Various reference 


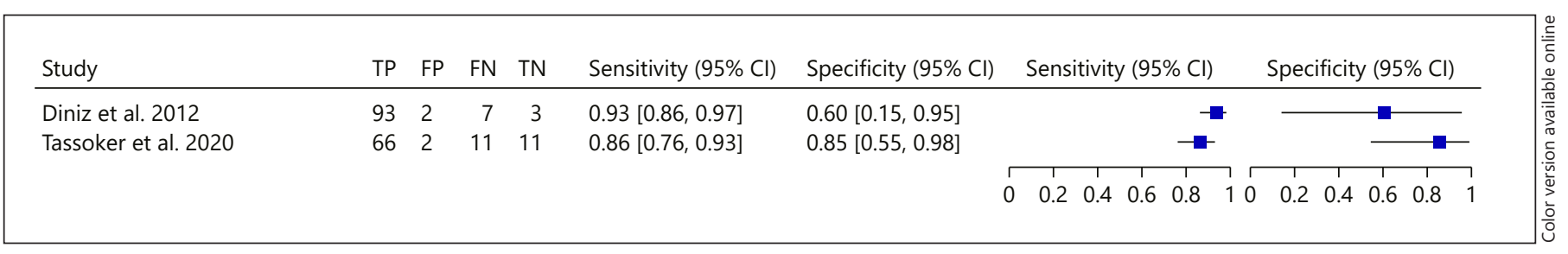

Fig. 4. Forest plot regarding ICDAS-II at D1 histological level.

standards were used for validation, with histological validation and operative intervention being used in 6 and 6 studies, respectively. Others included BW, computed microtomography, and visual examination (in some cases after the removal of orthodontic brackets or the use of elastic orthodontic separators) and were also relatively evenly represented. Se of the DD Pen ranged from 0.16 to 1 and Sp from 0.2 to 1 . While these ranges are broadly similar to corresponding ranges of the $\mathrm{DD}$, there were some differences. Az for the DD Pen ranged from 0.4 to 0.95. Again, there was a variety of cut-off points used for the calibration of the device (Tables 2, 3; online suppl. Tables 2-4).

\section{CarieScan Pro}

The CarieScan Pro device was evaluated in 5 studies, with a considerably smaller number of teeth assessed, namely, 25-120 across studies, but similarly reporting duplicate examination screenings, as per the number of investigators involved. Histological validation was used as the reference standard in 3 studies and operative intervention in 1. Se of the CarieScan Pro device ranged from 0.72 to 0.91 , Sp from 0 to 0.92 , and Az from 0.47 to 0.92 (Tables 2, 3; online suppl. Tables 2-4).

\section{VistaProof}

The VistaProof fluorescence camera was evaluated in 4 studies, with the number of teeth assessed at 32-619, again aligning with duplicate examination schemes. Histological validation was used as the reference standard in 1 study and, similarly, the operative intervention was used as the reference standard in another. Se of the VistaProof fluorescence camera ranged from 0.26 to 0.92 and $S p$ from 0.41 to $0.98 ; \mathrm{Az}$ was available in only 1 study and stood at 0.66-0.97 (Tables 2, 3; online suppl. Tables 2-4).

\section{SoproLife}

The SoproLife fluorescence camera as a diagnostic modality was assessed in 4 studies. Sample sizes ranged between 37 and 433 teeth, and the examination procedure was done in triplicate. Histological validation was used as the reference standard in 1 study and visual examination (ICDAS-II) for the rest. Se of the SoproLife fluorescence camera ranged from 0.86 to 0.98 , Sp from 0.55 to 0.96 , and $\mathrm{Az}$ from 0.89 to 0.98 (Tables 2, 3; online suppl. Tables 2-4).

\section{Miscellaneous Methods}

Various diagnostic modalities, including OCT, NIR, quantitative light-induced fluorescence (QLF), and modifications and combinations of the methods described above, were evaluated in 19 studies. Representation of these methods was sparse with none of them being assessed in $>2$ studies. There was a broad range of sample sizes $(25-1,160$ teeth), as expected when considering the heterogeneity of the diagnostic tools (online suppl. Table 5).

Effect of Index Test, Meta-Analyses, Additional Analyses

Out of the 51 articles included in the qualitative analysis, 5 were included in the quantitative synthesis, following the appraisal of the heterogeneity of study conditions and settings. All the studies included in the quantitative synthesis examined permanent teeth. A pooled estimate, resulting from formal statistical analysis was only possible for the DD, due to the low number of studies included for the other comparisons (i.e., <4). For all studies included in the quantitative synthesis, the reference standard adopted was either histological examination or operative intervention, the surfaces were occlusal, and the dentition was permanent.

\section{ICDAS-II Visual System}

Two studies investigating the diagnostic performance of ICDAS-II at the D1 histological level were included in the quantitative synthesis (Fig. 4; online suppl. Fig. 1). Diniz et al. [2012] reported Se of 0.93 (95\% CI: 0.86, 0.97) and Sp of 0.60 (95\% CI: 0.15, 0.95). In a more recent study [Tassoker et al., 2020], the corresponding values stood at $0.86(95 \%$ CI: $0.76,0.93)$ and 0.85 (95\% CI: $0.55,0.98)$, respectively.

\section{Ekstrand's Visual System}

Three studies examined the diagnostic performance of Ekstrand's visual criteria at the D3 histological level and 


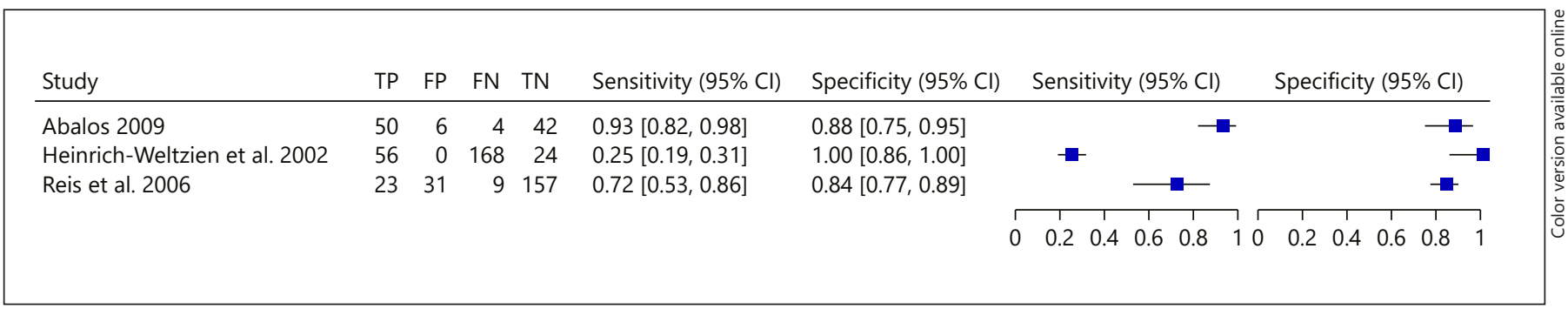

Fig. 5. Forest plot regarding Ekstrand's system at D3 histological level.

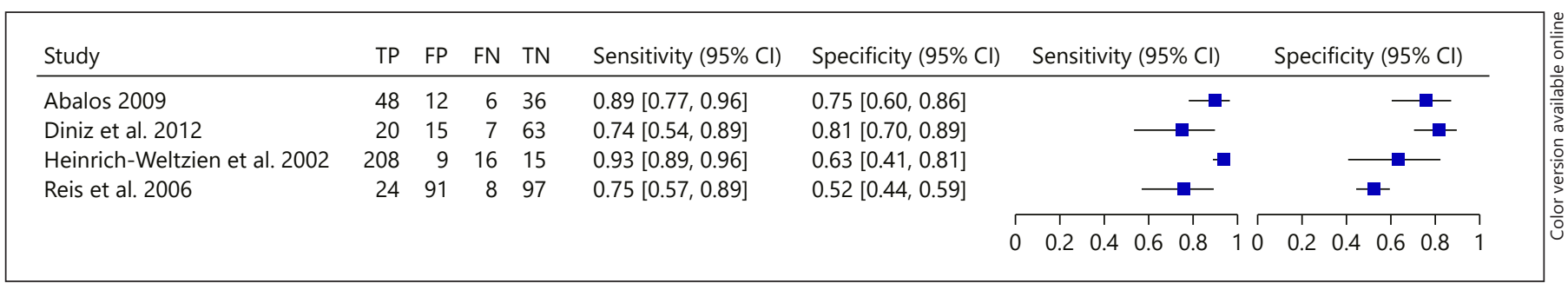

Fig. 6. Forest plot regarding DIAGNOdent at D3 histological level.

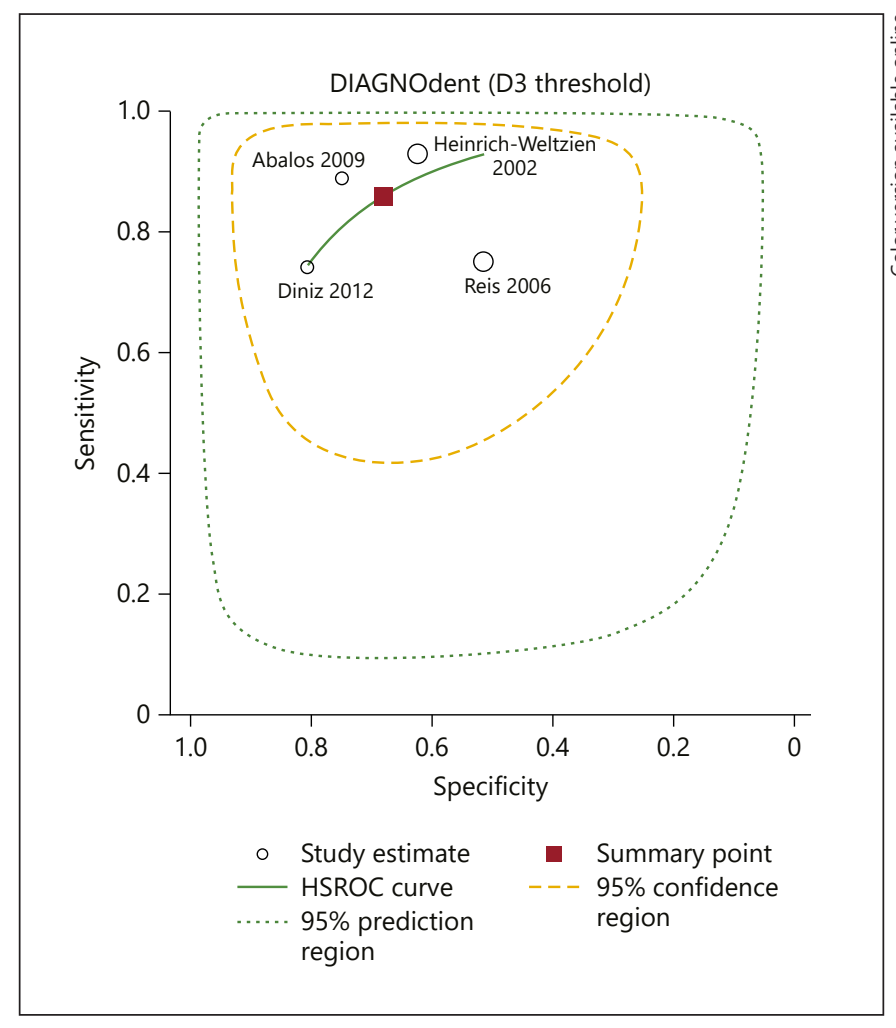

Fig. 7. HSROC curve regarding DIAGNOdent at D3 histological level. they were synthesized (Fig. 5; online suppl. Fig. 2). Abalos et al. [2009] showed Se of 0.93 (95\% CI: 0.82, 0.98) and Sp of 0.88 (95\% CI: 0.75, 0.95), while Reis et al. [2006] reported 0.72 (95\% CI: 0.53-0.86) and 0.84 (95\% CI: 0.77, 0.89), respectively. Furthermore, Heinrich-Weltzien et al. 흥 [2002] estimated a considerably lower Se of 0.25 (95\% CI: $0.19,0.31$ ), while the $\mathrm{Sp}$ was at similar levels to the other 2 studies, i.e., 1.00 (95\% CI: 0.86, 1.00).

\section{DIAGNOdent 2095}

Four studies [Heinrich-Weltzien et al., 2002; Reis et al., 2006; Abalos et al., 2009; Diniz et al., 2012] reported on the diagnostic performance of $\mathrm{DD}$ at the $\mathrm{D} 3$ histological level and were included in the meta-analysis. The breakdown of individual studies for both Se and Sp is presented in Figure 6. The HSROC curve is also presented, while the summary estimate for Se was 0.86 (95\% CI: 0.75, 0.92), while the Sp values were lower at 0.68 (95\% CI: 0.54, 0.79) (Fig. 7). The overall DOR, as a single measure for diagnostic effectiveness and performance of DD was 13.0 (95\% CI: 5.25, 32.10), revealing 13 times higher odds for DD being positive in identifying true caries, compared to being positive when no true caries exist.

DD Pen

Two studies exploring the diagnostic performance of DD Pen at the D1 histological level were included (Fig. 8; 


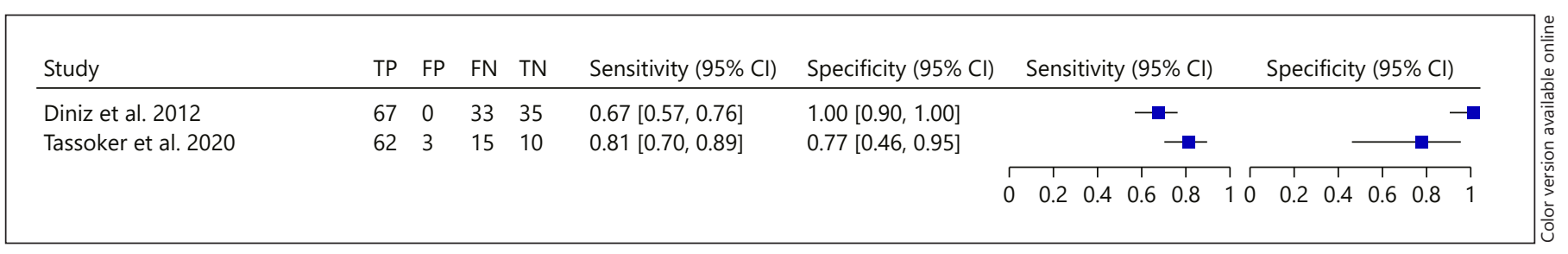

Fig. 8. Forest plot regarding DIAGNOdent Pen at D1 histological level.

online suppl. Fig. 3). Diniz et al. [2012] reported Se 0.67 ( $95 \%$ CI: $0.57,0.76)$ and Sp of $1(95 \%$ CI: $0.9,1)$, while the recent study of Tassoker et al. [2020] calculated respective values of 0.81 (95\% CI: 0.70-0.89) and 0.77 (95\% CI: 0.46, 0.95).

No additional analysis, sensitivity analysis, or exploration for publication bias could be performed due to the paucity of studies included in the quantitative synthesis.

\section{Discussion}

\section{Summary of the Evidence}

Visual examination for the detection of caries is the most common diagnostic method, mainly due to its costeffectiveness. Even though the ranges reported in this study appear relatively wide, most studies suggest moderate-to-high Se and Sp values. Regarding the Se of visual methods for the occlusal surfaces of permanent teeth, the study by Heinrich Weltzien et al. [2002] constitutes a significant outlier at only 0.25 , and the second-lowest Se value was 0.54 . According to their study, visual examination could not correctly identify all occlusal caries and a considerable amount remained undetected. BW, as a supplement to visual examination, is one of the oldest methods for caries detection. Most of the included studies indicated a low Se in the detection of incipient caries as well as in most occlusal lesions without signs of cavitation. Conversely, high $\mathrm{Sp}$ values were reported across all types of surfaces and dentitions, thus indicating the potential of this well-established tool to streamline and correctly classify non- carious surfaces.

The DD is one of the most thoroughly studied devices in the contemporary scientific literature. The original DD can only be used on occlusal surfaces. The Se and Sp values in the individual studies ranged from moderate to high in both permanent and primary teeth. The original DD has been superseded by the DD Pen, a more compact and updated version. The latter may be used for the detection of approximal carious lesions, unlike its predecessor. In terms

Detection Methods for Early Caries

Diagnosis of Se, several studies [Diniz et al., 2012, Cinar et al., 2013, Kucukyilmaz et al., 2015, Kockanat and Unal, 2017] report a modest increase in occlusal caries detection compared to with the original DD. On the other hand, Sp values seemed to be similar or even slightly lower than the corresponding values obtained with the original DD which is still in use. For approximal lesions, the Se values for permanent teeth and the $S p$ values for primary teeth were fairly high. However, the Se values for primary teeth and the Sp values for permanent teeth varied across studies.

The VistaProof fluorescence camera appears to have moderate-to-high Sp on buccal and occlusal surfaces. Highly variable Se values for occlusal lesions, in conjunction with the very limited number of studies assessing VistaProof in a clinical setting, preclude any further conclusion or speculation regarding this tool. A similar picture is formulated for the CariesScan Pro device, thus, imposing further uncertainty for clinical practice decision-making based on the existing evidence. The SoproLife fluorescence camera appears promising for both permanent and primary dentition, firstly, regarding its potential to correctly identify occlusal carious lesions, and secondly, as it does not seem to considerably lag where Sp is concerned. Further assessment of this tool would be useful. The QLF system was assessed in only 2 of the studies included in this review, although it is a diagnostic device that is relatively well-studied in the scientific literature, mostly in an in vitro setting. To date, it appears that the QLF system has been studied with sound emphasis on its technical aspects rather than its diagnostic validity in a clinical setting. This is possibly one of the main reasons why the QLF system appears under-represented in this review. Additional, well-designed clinical trials for the performance of QLF are necessary to provide clinically relevant information.

\section{Findings in Context}

Previous systematic reviews in the field of diagnostic tools for early caries detection do exist. Gomez et al. [2013] found a large variation and lack of consistency regarding Se and Sp values across the analytical tools exam- 
ined in both in vitro and in vivo studies with no distinction between the DD and DD Pen. However, since the publication of their report, new original studies have been published, to timely substantiate an update in the search for existing evidence, with our systematic review identifying a surplus of approximately 24 in vivo (clinical) studies. Other reviews have appeared recently in the literature, but with a scope to provide evidence for a limited number of specific analytical techniques and lacking a clear discrimination between updated tools such as the DD Pen and its predecessor, or between different types of tooth surfaces, or considering only certain specific reference standards [Iranzo-Cortés et al., 2019].

The studies contributing to this review present, in many cases, considerably variable results. This pertains largely to the heterogeneity inherent in the different methodologies followed in the individual studies, and subsequently reflects the overall heterogeneity in the results of meta-analyses. This is endemic to medical research [Alba et al., 2016; Rhodes et al., 2018]. A recent report on orthodontic meta-analyses from Cochrane and non-Cochrane reviews, revealed that more than onethird of the reviews presented substantial levels of heterogeneity [Koletsi et al., 2018]. Speculations in this respect may relate to different cut-off points or different techniques for the interpretation of the diagnostic methods. Moreover, the assessment of carious lesions of various stages across clinical studies might also be liable for the considerable heterogeneity present in the results of this study. Differences in the experimental design of the included studies, especially in the reference standards used, could clearly have affected the outcomes. Clearly, more randomized controlled trials, with comparable protocols, experimental designs, and outcomes, are to be welcomed and valued.

Finding the ideal early caries diagnostic method appears a somewhat utopian notion, in view of the considerable variation in Se and Sp values and lack of Az values in many studies. A dynamic equilibrium of the false-positive and false-negative rates in diagnosis should be anticipated as the most effective means. Generally, on occlusal surfaces, the performance of visual methods and the DIAGNOdent devices appears similar, with BW lagging behind. On approximal surfaces of primary teeth, the DD Pen appears to be more valid than BW, with the added benefit of not emitting ionizing radiation.

\section{Strengths and Limitations}

To the best of our knowledge, this is, to date, the first comprehensive systematic review and meta-analysis of clinical trials assessing all available diagnostic methods. Previous studies on this topic typically included in vitro studies in their analysis, thus making the findings potentially less clinically relevant. A comprehensive assessment of all available diagnostic means for non-cavitated carious lesions was performed, based on a concise literature search of all the published and unpublished literature recorded in 5 databases. The protocol of this review was a priori registered and followed a pre-defined methodology, one that safeguarded against selective reporting and considerable deviations from the original design [Fleming et al., 2015; Koufatzidou et al., 2019].

On the other hand, some limitations do exist. More than $60 \%$ of the included studies were characterized as having a high risk of bias regarding the reference standard, which can be attributed to the strict eligibility criteria used. Specifically, we judged studies as having a low risk of bias only when histological examination (considered the gold standard) was reported as the reference standard. However, it should be noted that a reference standard such as histology applied to ex vivo studies can only be performed in clinical studies to a relatively small subset of teeth that can be extracted and processed in the laboratory. This brings a non-negligible source of bias, i.e., the selection of teeth (and patients) where extraction is scheduled for specific treatments (third molars, deciduous teeth prior to natural shedding, and periodontal or orthodontic patients) to comply with ethical considerations. Operative intervention through drilling to identify the carious lesions was also considered a valid reference standard and, in fact, a close counterpart of histologic examination for the quantitative synthesis. It was the second most frequent validation method after histological examination and was used in 13 studies, with publication dates scattered over a wide time range. As a result, there have been different definitions for the term "early" or "incipient" caries over the years as well as various approaches for the management of these lesions. It should also be noted that teeth with obvious cavities were not included and, in this way, teeth with clinically important lesions were not taken into consideration, as per the reviews' aims, so false-negatives do exist. For instance, an occlusal carious lesion classified as ICDAS 3 is characterized as "early," but in some cases the appropriate management is operative intervention (e.g., fissurotomy, enameloplasty, etc.). Consequently, a proportion, at least, of early carious lesions do fit the aim of our study and present themselves as an "operative intervention" reference standard.

In addition, approximately two-thirds of the included studies were considered to have an unclear risk of bias 
in the domain of patient selection. This percentage agrees with the findings of another systematic review and meta-analysis that also used the QUADAS-2 tool [Iranzo-Cortés et al., 2019]. As previously discussed, the clear heterogeneity between individual study reports did not allow for many studies to be mathematically synthesized and the results to benefit the precision of the summary effect. On similar grounds, predefined additional analyses or publication bias effects could not be formally explored.

The fact that only 5 studies could be included in the meta-analysis due to the scarcity of data, despite considerable efforts to retrieve the necessary data from the authors, is also an important limitation of this study. Concerning the personal communication with the authors, 27 individualized messages were sent for providing data. Eighteen authors did not answer, even after reminders were sent twice after a pre-specified time interval of 2 and then a further 4 weeks. Four responded but they did not provide the data needed, even after specific queries and reminders, 2 replied that they no longer had access to the data, 1 had gone into retirement, and another provided a different dataset from the one we requested. All conversations were recorded in a standardized format but cannot be accessed due to the GDPR policy.

\section{Conclusions}

Although many diagnostic methods have emerged and studied thoroughly in recent years, robust conclusions cannot be drawn regarding their definite effectiveness. The results of this systematic review and meta-analysis reveal a considerable variation in the Se and $S p$ values detected, due to the heterogeneity in the experimental settings of individual studies.

However, based on the results of this report, clinical considerations may be framed in terms of the most-valued diagnostic use of the existing tools, per tooth and surface:
- Visual examination remains the method of choice across all types of surfaces and dentitions, irrespective of the visual criteria used.

- In permanent teeth, visual examination may be supplemented by DD for the diagnosis of occlusal caries. When examining approximal surfaces, visual examination may be enhanced by BW.

- In primary teeth, visual examination may be supplemented with the DD Pen for the diagnosis of occlusal lesions. For approximal surfaces, the DD Pen may also be used as an adjunct to visual examination.

\section{Acknowledgements}

We would like to thank Michele Diniz, Daniel Fried, Melek Tassoker, and Fausto Medeiros Mendes for providing the necessary statistical data from their publications to proceed with the quantitative synthesis.

\section{Conflict of Interest Statement}

The authors have no conflicts of interest to declare.

\section{Funding Sources}

The authors did not receive any funding.

\section{Author Contributions}

P.F. drafted the protocol, searched for trials, obtained full texts, inserted data into Zotero, evaluated trials to include, extracted data from trials, entered data into Review Manager, communicated with authors, carried out the analysis, and drafted the final review. E.O. drafted the protocol, searched for trials, obtained full texts, entered data into Zotero, evaluated trials to include, extracted data from trials, carried out the analysis, and drafted the final review. D.K. drafted the protocol, entered data into Review Manager, carried out the analysis, interpreted the analysis, and drafted the final review. C.R. drafted the protocol, developed a search strategy, selected which trials to include, and drafted the final review.

\section{References}

Abalos C, Herrera M, Jiménez-Planas A, Llamas R. Performance of laser fluorescence for detection of occlusal dentinal caries lesions in permanent molars: an in vivo study with total validation of the sample. Caries Res. 2009;43(2):137-41.

Akbari M, Ahrari F, Jafari M. A comparative evaluation of DIAGNOdent and caries detector dye in detection of residual caries in prepared cavities. J Contemp Dent Pract. 2012 Jul;13(4):515-20.

Detection Methods for Early Caries Diagnosis
Alba AC, Alexander PE, Chang J, MacIsaac J, DeFry S, Guyatt GH. High statistical heterogeneity is more frequent in meta-analysis of continuous than binary outcomes. J Clin Epidemiol. 2016 Feb;70:129-35.

Angnes V, Angnes G, Batisttella M, Grande RH, Loguercio AD, Reis A. Clinical effectiveness of laser fluorescence, visual inspection and radiography in the detection of occlusal caries. Caries Res. 2005 Nov-Dec;39(6):490-5.
Bader JD, Shugars DA, Bonito AJ. A systematic review of the performance of methods for identifying carious lesions. J Public Health Dent. 2002;62(4):201-13.

Baelum V, Hintze H, Wenzel A, Danielsen B, Nyvad B. Implications of caries diagnostic strategies for clinical management decisions. Community Dent Oral Epidemiol. 2012 Jun;40(3):257-66. 
Bahrololoomi Z, Ezoddini F, Halvani N. Comparison of Radiography, Laser Fluorescence and Visual Examination for Diagnosing Incipient Occlusal Caries of Permanent First Molars. J Dent (Tehran). 2015 May;12(5):324-32.

Barbería E, Maroto M, Arenas M, Silva CC. A clinical study of caries diagnosis with a laser fluorescence system. J Am Dent Assoc. 2008 May;139(5):572-9.

Bizhang M, Wollenweber N, Singh-Hüsgen P, Danesh G, Zimmer S. Pen-type laser fluorescence device versus bitewing radiographs for caries detection on approximal surfaces. Head Face Med. 2016 Nov;12(1):30.

Błażejewska A, Dacyna N, Niesiobędzki P, Trzaska M, Gozdowski D, Turska-Szybka A, et al. Comparison of the detection of proximal caries in children and youth using DIAGNO$\mathrm{cam}^{\circledR}$ and bitewing radiovisiography. Dent Med Probl. 2016;53(4):468-75.

Bozdemir E, Karaarslan ES, Ozsevik AS, Ata Cebe $\mathrm{M}, \mathrm{Aktan} \mathrm{AM}$. In vivo performance of two devices for occlusal caries detection. Photomed Laser Surg. 2013 Jul;31(7):322-7.

Braga MM, Ekstrand KR, Martignon S, Imparato JC Ricketts DN, Mendes FM. Clinical performance of two visual scoring systems in detecting and assessing activity status of occlusal caries in primary teeth. Caries Res. 2010;44(3):300-8.

Bussaneli DG, Restrepo M, Boldieri T, Albertoni TH, Santos-Pinto L, Cordeiro RC. Proximal caries lesion detection in primary teeth: does this justify the association of diagnostic methods? Lasers Med Sci. 2015 Dec;30(9):2239-44.

Castilho LS, Cotta FV, Bueno AC, Moreira AN, Ferreira EF, Magalhães CS. Validation of DIAGNOdent laser fluorescence and the International Caries Detection and Assessment System (ICDAS) in diagnosis of occlusal caries in permanent teeth: an in vivo study. Eur J Oral Sci. 2016 Apr;124(2):188-94.

Chan M, Dadul T, Langlais R, Russell D, Ahmad M. Accuracy of extraoral bite-wing radiography in detecting proximal caries and crestal bone loss. J Am Dent Assoc. 2018;149(1):51-8.

$\mathrm{Chu} \mathrm{CH}$, Lo EC, You DS. Clinical diagnosis of fissure caries with conventional and laser-induced fluorescence techniques. Lasers Med Sci. 2010 May;25(3):355-62.

Cınar C, Atabek D, Odabaş ME, Olmez A. Comparison of laser fluorescence devices for detection of caries in primary teeth. Int Dent $J$. 2013 Apr;63(2):97-102.

Costa AM, Paula LM, Bezerra AC. Use of Diagnodent for diagnosis of non-cavitated occlusal dentin caries. J Appl Oral Sci. 2008 JanFeb;16(1):18-23.

Coutinho TC, daRocha Costa C. An in vivo comparison of radiographic and clinical examination with separation for assessment of approximal caries in primary teeth. Eur J Paediatr Dent. 2014 Dec;15(4):371-4.

de Souza LA, Cancio V, Tostes MA. Accuracy of pen-type laser fluorescence device and radiographic methods in detecting approximal carious lesions in primary teeth - an in vivo study. Int J Paediatr Dent. 2018 Jul;28(5):472-80.
Diniz MB, Boldieri T, Rodrigues JA, Santos-Pinto L, Lussi A, Cordeiro RCL. The performance of conventional and fluorescence-based methods for occlusal caries detection: an in vivo study with histologic validation. J Am Dent Assoc. 2012;143(4):339-50.

Diniz MB, Cordeiro RC, Ferreira-Zandona AG. Detection of Caries Around Amalgam Restorations on Approximal Surfaces. Oper Dent. 2016 Jan-Feb;41(1):34-43.

Ekstrand KR, Qvist V. The impact of a national caries strategy in Greenland after 4 years. Int J Paediatr Dent. 2015 Jul;25(4):255-66.

Ekstrand KR, Ricketts DN, Longbottom C, Pitts NB. Visual and tactile assessment of arrested initial enamel carious lesions: an in vivo pilot study. Caries Res. 2005 May-Jun;39(3):173-7.

Fejerskov O. Concepts of dental caries and their consequences for understanding the disease. Community Dent Oral Epidemiol. 1997 Feb;25(1):5-12.

Fleming PS, Koletsi D, Dwan K, Pandis N. Outcome discrepancies and selective reporting: impacting the leading journals? PLoS One. 2015 May;10(5):e0127495.

Freitas LA, Santos MT, Guaré RO, Lussi A, Diniz MB. Association Between Visual Inspection, Caries Activity Status, and Radiography with Treatment Decisions on Approximal Caries in Primary Molars. Pediatr Dent. 2016 MarApr;38(2):140-7.

Gimenez T, Piovesan C, Braga MM, Raggio DP, Deery C, Ricketts DN, et al. Visual Inspection for Caries Detection: A Systematic Review and Meta-analysis. J Dent Res. 2015 Jul;94(7):895-904.

Goel A, Chawla HS, Gauba K, Goyal A. Comparison of validity of DIAGNOdent with conventional methods for detection of occlusal caries in primary molars using the histological gold standard: an in vivo study. J Indian Soc Pedod Prev Dent. 2009 Oct-Dec;27(4):227-34.

Gomez J. Detection and diagnosis of the early caries lesion. BMC Oral Health. 2015;15 Suppl 1(Suppl 1):S3-S3

Gomez J, Tellez M, Pretty IA, Ellwood RP, Ismail AI. Non-cavitated carious lesions detection methods: a systematic review. Community Dent Oral Epidemiol. 2013 Feb;41(1):54-66.

Goodwin M, Sanders C, Davies G, Walsh T, Pretty IA. Issues arising following a referral and subsequent wait for extraction under general anaesthetic: impact on children. BMC Oral Health. 2015 Jan;15(1):3.

Heinrich-Weltzien R, Weerheijm KL, Kuhnisch J, Oehme T, Stosser L. Clinical evaluation of visual, radiographic, and laser fluorescence methods for detection of occlusal caries. ASDC J Dent Child. 2002 Aug;69(2):127-32.

Hintze H, Wenzel A, Danielsen B, Nyvad B. Reliability of visual examination, fibre-optic transillumination, and bite-wing radiography, and reproducibility of direct visual examination following tooth separation for the identification of cavitated carious lesions in contacting approximal surfaces. Caries Res. 1998;32(3):204-9.
Holtzman JS, Kohanchi D, Biren-Fetz J, Fontana M, Ramchandani M, Osann K, et al. Detection and proportion of very early dental caries in independent living older adults. Lasers Surg Med. 2015 Nov;47(9):683-8.

Huth KC, Lussi A, Gygax M, Thum M, Crispin A, Paschos E, et al. In vivo performance of a laser fluorescence device for the approximal detection of caries in permanent molars. J Dent. 2010 Dec;38(12):1019-26.

Huth KC, Neuhaus KW, Gygax M, Bücher K, Crispin A, Paschos E, et al. Clinical performance of a new laser fluorescence device for detection of occlusal caries lesions in permanent molars. J Dent. 2008 Dec;36(12):103340.

Iranzo-Cortés JE, Montiel-Company JM, Almerich-Torres T, Bellot-Arcís C, Almerich-Silla JM. Use of DIAGNOdent and VistaProof in diagnostic of Pre-Cavitated Caries Lesions-A Systematic Review and Meta-Analysis. J Clin Med. 2019 Dec;9(1):E20.

Jablonski-Momeni A, Heinzel-Gutenbrunner M, Klein SM. In vivo performance of the VistaProof fluorescence-based camera for detection of occlusal lesions. Clin Oral Investig. 2014 Sep;18(7):1757-62.

Jones CM, Davies GM, Monaghan N, Morgan MZ, Neville JS, Pitts NB. The caries experience of 5-year-old children in Scotland in 2013-2014, and in England and Wales in 2014-2015. Reports of cross-sectional dental surveys using BASCD criteria. Community Dent Health. 2017 Sep;34(3):157-62.

Jung E-H, Lee E-S, Jung H-I, Kang S-M, de Josselin de Jong E, et al. Development of a fluorescence-image scoring system for assessing noncavitated occlusal caries. Photodiagnosis Photodyn Ther. 2018 Mar;21:36-42.

Kassebaum NJ, Bernabé E, Dahiya M, Bhandari B, Murray CJ, Marcenes W. Global burden of untreated caries: a systematic review and metaregression. J Dent Res. 2015 May;94(5):650-8.

Kavvadia K, Lagouvardos P. Clinical performance of a diode laser fluorescence device for the detection of occlusal caries in primary teeth. Int J Paediatr Dent. 2008 May;18(3):197-204.

Kavvadia K, Seremidi K, Reppa C, Makou M, Lagouvardos P. Validation of fluorescence devices for evaluation of white spot lesions in orthodontic patients. Eur Arch Paediatr Dent. 2018 Apr;19(2):83-9.

Kim E-S, Lee E-S, Kang S-M, Jung E-H, de Josselin de Jong E, Jung H-I, et al. A new screening method to detect proximal dental caries using fluorescence imaging. Photodiagnosis Photodyn Ther. 2017;20:257-62.

Kockanat $A$, Unal $M$. In vivo and in vitro comparison of ICDAS II, DIAGNOdent pen, CarieScan PRO and SoproLife camera for occlusal caries detection in primary molar teeth. Eur J Paediatr Dent. 2017 Jun;18(2):99-104.

Koletsi D, Fleming PS, Michelaki I, Pandis N. Heterogeneity in Cochrane and non-Cochrane meta-analyses in orthodontics. J Dent. 2018;74:90-4. 
Koufatzidou M, Koletsi D, Fleming PS, Polychronopoulou A, Pandis N. Outcome reporting discrepancies between trial entries and published final reports of orthodontic randomized controlled trials. Eur J Orthod. 2019 May;41(3):225-30.

Krause F, Jepsen S, Braun A. Comparison of two laser fluorescence devices for the detection of occlusal caries in vivo. Eur J Oral Sci. 2007 Aug;115(4):252-6.

Kucukyilmaz E, Sener Y, Botsali MS. In Vivo and In Vitro performance of Conventional Methods, DIAGNOdent, and an Electronic Caries Monitor for Occlusal Caries Detection in Primary Teeth. Pediatr Dent. 2015 JulAug;37(4):E14-22.

Lenzi TL, Piovesan C, Mendes FM, Braga MM, Raggio DP. In vitro performance of QLF system and conventional methods for detection of occlusal caries around tooth-colored restorations in primary molars. Int $\mathrm{J}$ Paediatr Dent. 2016 Jan;26(1):26-34.

Lino JR, Ramos-Jorge J, Coelho VS, Ramos-Jorge ML, Moysés MR, Ribeiro JC. Association and comparison between visual inspection and bitewing radiography for the detection of recurrent dental caries under restorations. Int Dent J. 2015 Aug;65(4):178-81.

Lussi A, Megert B, Longbottom C, Reich E, Francescut $\mathrm{P}$. Clinical performance of a laser fluorescence device for detection of occlusal caries lesions. Eur J Oral Sci. 2001 Feb;109(1):14-9.

Matalon S, Feuerstein O, Calderon S, Mittleman A, Kaffe I. Detection of cavitated carious lesions in approximal tooth surfaces by ultrasonic caries detector. Oral Surg Oral Med Oral Pathol Oral Radiol Endod. 2007 Jan;103(1):109-13.

McInnes MD, Moher D, Thombs BD, McGrath TA, Bossuyt PM, Clifford T, et al.; and the PRISMA-DTA Group. Preferred Reporting Items for a Systematic Review and Meta-analysis of Diagnostic Test Accuracy Studies: the PRISMA-DTA Statement. JAMA. 2018 Jan;319(4):388-96.

Melo M, Pascual A, Camps I, Del Campo Á. In vivo study of different methods for diagnosing pit and fissure caries. J Clin Exp Dent. 2015 Jul;7(3):e387-91.

Menem R, Barngkgei I, Beiruti N, Al Haffar I, Joury E. The diagnostic accuracy of a laser fluorescence device and digital radiography in detecting approximal caries lesions in posterior permanent teeth: an in vivo study. Lasers Med Sci. 2017 Apr;32(3):621-8.
Mortensen D, Hessing-Olsen I, Ekstrand KR, Twetman S. In-vivo performance of impedance spectroscopy, laser fluorescence, and bitewingradiographs for occlusal caries detection. Quintessence Int. 2018;49(4):293-9.

Newman B, Seow WK, Kazoullis S, Ford D, Holcombe T. Clinical detection of caries in the primary dentition with and without bitewing radiography. Aust Dent J. 2009 Mar;54(1):2330.

Novaes TF, Matos R, Braga MM, Imparato JC, Raggio DP, Mendes FM. Performance of a pen-type laser fluorescence device and conventional methods in detecting approximal caries lesions in primary teeth -in vivo study. Caries Res. 2009;43(1):36-42.

Ozkan G, Guzel KG. Clinical evaluation of nearinfrared light transillumination in approximal dentin caries detection. Lasers Med Sci. 2017 Aug;32(6):1417-22.

Rechmann P, Charland D, Rechmann BM, Feath erstone JD. Performance of laser fluorescence devices and visual examination for the detection of occlusal caries in permanent molars. J Biomed Opt. 2012 Mar;17(3):036006.

Reis A, Mendes FM, Angnes V, Angnes G, Grande RH, Loguercio AD. Performance of methods of occlusal caries detection in permanent teeth under clinical and laboratory conditions. J Dent. 2006 Feb;34(2):89-96.

Rhodes KM, Turner RM, Savović J, Jones HE, Mawdsley D, Higgins JP. Between-trial heterogeneity in meta-analyses may be partially explained by reported design characteristics. J Clin Epidemiol. 2018;95:45-54.

Ribeiro AA, Purger F, Rodrigues JA, Oliveira PR, Lussi A, Monteiro AH, et al. Influence of contact points on the performance of caries detection methods in approximal surfaces of primary molars: an in vivo study. Caries Res. 2015;49(2):99-108.

Rocha RO, Ardenghi TM, Oliveira LB, Rodrigues $\mathrm{CR}$, Ciamponi AL. In vivo effectiveness of laser fluorescence compared to visual inspection and radiography for the detection of occlusal caries in primary teeth. Caries Res. 2003 Nov-Dec;37(6):437-41.

Schardt C, Adams MB, Owens T, Keitz S, Fontelo P. Utilization of the PICO framework to improve searching PubMed for clinical questions. BMC Med Inform Decis Mak. 2007 Jun;7(1):16-16.

Simon JC, Lucas SA, Staninec M, Tom H, Chan $\mathrm{KH}$, Darling CL, et al. Near-IR transillumination and reflectance imaging at $1,300 \mathrm{~nm}$ and $1,500-1,700 \mathrm{~nm}$ for in vivo caries detection. Lasers Surg Med. 2016 Nov;48(9):828-36.
Singh R, Tandon S, Rathore M, Tewari N, Singh N, Shitoot AP. Clinical performance of ICDAS II, radiovisiography, and alternating current impedance spectroscopy device for the detection and assessment of occlusal caries in primary molars. J Indian Soc Pedod Prev Dent. 2016;34(2):152-8.

Souza JF, Boldieri T, Diniz MB, Rodrigues JA, Lussi A, Cordeiro RC. Traditional and novel methods for occlusal caries detection: performance on primary teeth. Lasers Med Sci. 2013 Jan;28(1):287-95.

Subka S, Rodd H, Nugent Z, Deery C. In vivo validity of proximal caries detection in primary teeth, with histological validation. Int J Paediatr Dent. 2019 Jul;29(4):429-38.

Tassoker M, Ozcan S, Karabekiroglu S. Occlusal Caries Detection and Diagnosis Using Visual ICDAS Criteria, Laser Fluorescence Measurements, and Near-Infrared Light Transillumination Images. Med Princ Pract. 2020;29(1):25-31.

Teo TK, Ashley PF, Louca C. An in vivo and in vitro investigation of the use of ICDAS, DIAGNOdent pen and CarieScan PRO for the detection and assessment of occlusal caries in primary molar teeth. Clin Oral Investig. 2014 Apr;18(3):737-44.

Theocharopoulou A, Lagerweij MD, van Strijp AJ. Use of the ICDAS system and two fluorescence-based intraoral devices for examination of occlusal surfaces. Eur J Paediatr Dent. 2015 Mar;16(1):51-5.

Vos T, Allen C, Arora M, Barber RM, Bhutta ZA, Brown A, et al.; GBD 2015 Disease and Injury Incidence and Prevalence Collaborators. Global, regional, and national incidence, prevalence, and years lived with disability for 310 diseases and injuries, 1990-2015: a systematic analysis for the Global Burden of Disease Study 2015. Lancet. 2016 Oct:388(10053):1545-602.

Whiting PF, Rutjes AW, Westwood ME, Mallett S, Deeks JJ, Reitsma JB, et al.; QUADAS-2 Group. QUADAS-2: a revised tool for the quality assessment of diagnostic accuracy studies. AnnInternMed.2011Oct;155(8):52936.

Zaidi I, Somani R, Jaidka S, Nishad M, Singh S, Tomar D. Evaluation of Different Diagnostic Modalities for Diagnosis of Dental Caries: An in vivo Study. Int $\mathrm{J}$ Clin Pediatr Dent. 2016;9(4):320-5.

Zeitouny M, Feghali M, Nasr A, Abou-Samra P, Saleh N, Bourgeois D, et al. SOPROLIFE system: an accurate diagnostic enhancer. ScientificWorldJournal. 2014;2014:924741. 